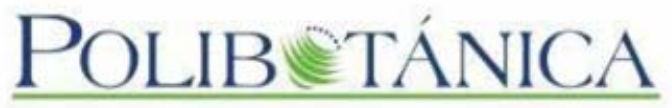

Polibotánica

ISSN electrónico: 2395-9525

polibotanica@gmail.com

Instituto Politécnico Nacional

México

http://www.polibotanica.mx

\title{
DESCRIPCIÓN DE ASPECTOS DEL SUELO Y COMPOSICIÓN FLORÍSTICA DEL HÁBITAT DE Lobelia villaregalis (CAMPANULACEAE), ESPECIE ENDÉMICA DE JALISCO, MÉXICO.
}

\section{DESCRIPTION OF SOIL AND FLORISTIC COMPOSITION OF THE HABITAT OF Lobelia villaregalis (CAMPANULACEAE), SPECIES ENDEMIC FROM JALISCO, MEXICO .}

Villa-Galaviz, E., L. Hernández-López, M. Harker y C. Neri-Luna. DESCRIPCIÓN DE ASPECTOS DEL SUELO Y COMPOSICIÓN FLORÍSTICA DEL HÁBITAT DE Lobelia villaregalis (CAMPANULACEAE), ESPECIE ENDÉMICA DE JALISCO, MÉXICO.

DESCRIPTION OF SOIL AND FLORISTIC COMPOSITION OF THE HABITAT OF Lobelia villaregalis (CAMPANULACEAE), SPECIES ENDEMIC FROM JALISCO, MEXICO.

\section{POLIBETÁNICA}

Instituto Politécnico Nacional
Núm. 49: 30-49 México. Enero 2020

DOI: 10.18387 polibotanica.49.3

(c) (i) Este es un artículo de acceso abierto bajo la licencia Creative Commons 4.0 Atribución-No Comercial (CC BY-NC 4.0 Internacional). 


\section{DESCRIPCIÓN DE ASPECTOS DEL SUELO Y COMPOSICIÓN FLORÍSTICA DEL HÁBITAT DE Lobelia villaregalis (CAMPANULACEAE), ESPECIE ENDÉMICA DE JALISCO, MÉXICO.}

\section{DESCRIPTION OF SOIL AND FLORISTIC COMPOSITION OF THE HABITAT OF Lobelia villaregalis (CAMPANULACEAE), SPECIES ENDEMIC FROM JALISCO, MEXICO.}

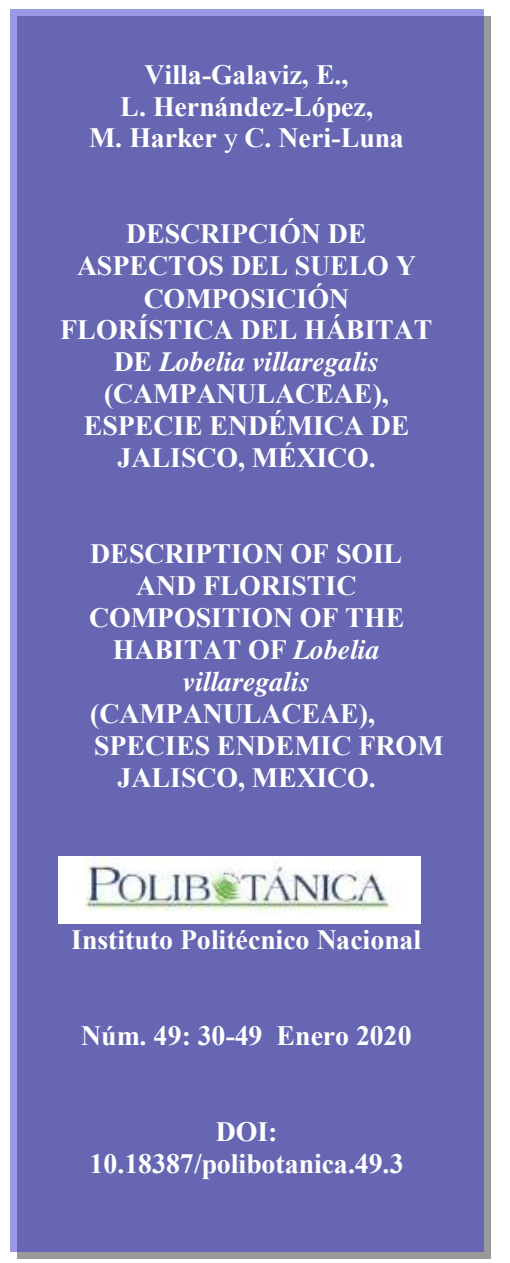

\begin{abstract}
E. Villa-Galaviz
Herbario Luz María Villarreal de Puga del Instituto de Botánica, Departamento de Botánica y Zoología; Laboratorio de Ecofisiología Vegetal, Departamento de Ecología, Centro Universitario de Ciencias Biológicas y Agropecuarias, Universidad de Guadalajara. Apartado postal 1-139, Zapopan 45101, Jalisco, México.

L. Hernández-López / leticia.hernandez@academicos.udg.mx

M. Harker

Herbario Luz María Villarreal de Puga del Instituto de Botánica, Departamento de Botánica y Zoología, Centro Universitario de Ciencias Biológicas y Agropecuarias, Universidad de Guadalajara. Apartado postal 1-139, Zapopan 45101, Jalisco, México.

C. Neri-Luna

Laboratorio de Ecofisiología Vegetal, Departamento de Ecología, Centro Universitario de Ciencias Biológicas y Agropecuarias, Universidad de Guadalajara. Apartado postal 1-139, Zapopan 45101, Jalisco, México.
\end{abstract}

RESUMEN: Lobelia villaregalis es una especie endémica de Jalisco, México. Desafortunadamente, se desconocen las características del hábitat donde se desarrolla, lo cual limita el establecimiento de estrategias de conservación, así como la determinación de la distribución potencial de la especie. En este estudio, se caracterizaron algunas propiedades del suelo, su asociación micorrícica y la vegetación circundante en dos localidades donde la planta crece. El suelo en ambas localidades es franco arenoso poco profundo $(1.5 \mathrm{~cm})$ y de baja fertilidad, la raíz de L. villaregalis mostró bajos porcentajes de colonización por hongos micorrícicos del tipo arbuscular. La comunidad vegetal corresponde al bosque mixto de encino-pino donde la diversidad de la flora asociada consta de 140 taxa incluidos en 102 géneros y 44 familias de plantas vasculares. Asteraceae incluye $32 \%$ de los taxa registrados, seguida de Poaceae y Pteridaceae (6\% y 5\% respectivamente). El estrato herbáceo es el más diverso (73\% de los taxa), seguido del arbóreo (13\%) y el arbustivo (11\%). De la riqueza total de especies, 31 (22.1\%) se comparten entre las dos localidades, 101 (72\%) se encuentran en "La Venta" siendo 70 exclusivas de este sitio, mientras que en "El Espinazo" se registran 70 taxa (50\%), de ellos 39 no se comparten. La composición florística del bosque mixto, así como las condiciones físicas de las localidades indican que $L$. villaregalis prefiere hábitats relativamente conservados con alta humedad y paredes o laderas pronunciadas con sustrato arenoso. Los resultados sugieren que la conservación de tales hábitats sería la estrategia adecuada para proteger la especie en cuestión.

Palabras clave: Lobelia, especie endémica, La Primavera, áreas protegidas, hongos micorrícicos arbusculares, conservación.

ABSTRACT: Lobelia villaregalis is a plant endemic to Jalisco, Mexico. The habitat where it grows is not well known and for this reason conservation strategies and the definition of potential distribution in other sites have not been defined. In this study a 
description of soil characteristics and floristics are presented for a ravine and a rock face where the plant grows. In both places, the soil is infertile, sandy loam only $1.5 \mathrm{~cm}$ deep where the roots present low percentages of colonization by arbuscular mycorrhizal fungi. Mixed pine-oak forest occurs in the two sites. The vascular flora includes 140 taxa in 102 genera and 44 families. Asteraceae is the most abundant representing 32\% of the taxa, followed by Poaceae and Pteridaceae (with 6\% and 5\% respectively). Herbs dominate the flora including $73 \%$ of the taxa followed by trees (13\%) and then shrubs (11\%). With respect to the total richness, 31 species (22.1\%) are shared by the two locations while 101 (72\%) are known in "La Venta" of which 70 are exclusive to this location, while in "El Espinazo" 70 taxa (50\%) are known and 39 are not shared. The floristic composition of the mixed forest as well as the physical conditions of the localities indicate that L. villaregalis prefers relatively conserved habitats with high humidity and pronounced walls or slopes with sandy substrate. The results suggest that the conservation of such habitats would be the appropriate strategy to protect the species in question.

Key words: Lobelia, endemic species, La Primavera, protected areas, arbuscular mycorrhizal fungi, conservation.

\section{INTRODUCCIÓN}

La familia Campanulaceae incluye 14 géneros y 117 especies para México (Rzedowski, 2019). Esta familia comprende el género Lobelia que se caracteriza por ser casi cosmopolita con 423 especies en 18 secciones. La sección Stenotium presenta amplia distribución y es la de mayor diversidad de especies con 153 (Lammers, 2011); debido a la presencia de flores espolonadas esta sección se divide en especies espolonadas y no espolonadas. Las especies espolonadas de Lobelia crecen en áreas húmedas en bosques templados desde Estados Unidos hasta Costa Rica. En particular, 10 especies crecen en la vertiente del Pacífico de la Sierra Madre Occidental en México (Gutiérrez-Sánchez et al., 2018). Dentro de las especies espolonadas de la sección Stenotium se encuentra Lobelia villaregalis Ayers, una especie endémica del Área de Protección de Flora y Fauna del Bosque La Primavera (APFFLP), Jalisco. La especie se caracteriza por ser una hierba perenne de $15-20 \mathrm{~cm}$ de alto, con tallos decumbentes, hojas simples ovadas de 15-30 $\mathrm{mm}$ de largo, margen dentado; inflorescencia racemosa, pedúnculo de 5-7 cm; flores de color rosa, zigomorfas, simpétalas de 1-1.2 cm, con espolón cónico de 8.5-10 $\mathrm{mm}$ y apertura dorsal; estambres $5-5.5 \mathrm{~mm}$, filamentos blancos, connados y anteras azules, cuyo fruto es una capsula con semillas numerosas. Su distribución es particularmente restringida, de hecho cuando la especie fue descrita sólo se conocía una localidad con una población de 5 individuos (Ayers, 1987). Favorablemente, se han encontrado poblaciones en otras localidades dentro del APFFLP (Hernández-López, en proceso), sin embargo, se hallan cerca de senderos recreativos y asentamientos irregulares lo que amenaza su existencia. Desafortunadamente, no es posible establecer estrategias adecuadas para su conservación dado que se desconocen las características del hábitat donde se desarrolla.

La distribución y abundancia de las comunidades vegetales está determinada por diversos factores (bióticos y abióticos), entre los que destacan: los mecanismos de dispersión, capacidad de adaptación a nuevas condiciones del hábitat e interacciones con otras especies (Wiens y Donoghue, 2004). No obstante, se ha venido señalando que un factor importante para la distribución de las especies vegetales, así como para su posterior propagación, es el conocimiento de las características del suelo donde se desarrollan (Huerta-Martínez et al., 2014; 2012). Las propiedades físicas del suelo como temperatura, textura, humedad y cantidad de materia orgánica, ayudan a la formación de microhábitats específicos para las plantas, particularmente las especialistas (Brady y Weil, 2002). Además de las características fisicoquímicas del suelo, los aspectos biológicos del mismo determinan el microhábitat de las especies vegetales, donde dentro de las relaciones más significativas que establecen es la denominada simbiosis micorrícica, que es un fenómeno ecológico-evolutivo producto de la interacción entre hongos y plantas con el suelo y el ambiente atmosférico que les ha brindado entre otras cosas: estabilización del suelo, mejor acceso a agua y nutrientes, así como 
bioprotección (estrés hídrico, patógenos, contaminantes del suelo) y bioregulación (Neri-Luna y Villarreal-Ruiz, 2012; Smith y Read, 2008). Asimismo, la simbiosis micorrícica es importante para aumentar las probabilidades de supervivencia y establecimiento de las plántulas, lo cual es determinante para los programas de re-población de especies (Davidson et al., 2016).

En el APFFLP se han realizado numerosas investigaciones (Gallegos, 2011). En lo que a la flora se refiere, existen diversos estudios, algunos con énfasis en grupos taxonómicos particulares, de localidades específicas y otros sobre diversos grupos biológicos en toda el área. Entre ellos destacan los trabajos de Curiel (1988), Reyna Bustos (1989) y CONANP (2000), en este último se da cuenta de 961 especies, 419 géneros y 107 familias de plantas vasculares, donde Campanulaceae incluye cinco taxa, pero desafortunadamente no se lista a L. villaregalis.

Con el objetivo de contribuir al conocimiento biológico que ayude a la conservación de $L$. villaregalis, en este estudio se describen algunas de las condiciones ambientales de las dos localidades donde la especie se ha encontrado, en particular, se dan a conocer las características del suelo, su potencial relación con hongos micorrícicos y la composición florística asociada.

\section{MÉTODOS}

\section{Área de estudio}

El Bosque la Primavera fue declarado en 1980 como Área de Protección de Flora y Fauna (APFFLP) con una superficie de 30,500 ha. Se ubica en la parte central del estado de Jalisco, al oeste de la Zona Metropolitana de Guadalajara, entre las coordenadas extremas $103^{\circ} 28^{\prime}$ a $103^{\circ} 42^{\prime}$ de longitud oeste y $20^{\circ} 32^{\prime}$ a $20^{\circ} 44^{\prime}$ de latitud norte (CONANP, 2000). Abarca la superficie de cuatro municipios que en orden descendente por su cobertura en el área son: Zapopan, Tala, Tlajomulco y El arenal (fig. 1).

El APFFLP presenta un rango de elevación de los 1400 a 2200 m s.n.m y forma parte de la Sierra La Primavera, la cual fisiográficamente pertenece a una zona donde se traslapan el Eje Neovolcánico Transversal y la Sierra Madre Occidental. Desde el punto de vista geológico, la Sierra de La Primavera es, en términos técnicos, una caldera volcánica formada por erupciones de tobas y piroclastos (Valdivia Ornelas, 2018). Los procesos geológicos y geomorfológicos recientes en combinación con el enfriamiento de tobas y su erosión posterior, le confieren al relieve de La Primavera rasgos únicos que deberían considerarse como Geopatrimonio (González Torreros et al., 2018). Los tipos de suelos mejor representados son regosol derivado del intemperismo de la toba, pómez y riolita, y litosol, resultante de procesos erosivos, los cuales cubren el $92 \%$ y $8 \%$ del área, respectivamente (CONANP, 2000). Las comunidades vegetales registradas, con base en la clasificación de Rzedowski (2006), son: bosque de encino (Quercus), bosque de encino-pino (Quercus-Pinus), bosque de pino (Pinus), bosque tropical caducifolio, vegetación riparia y vegetación secundaria o ruderal (Reyna Bustos, 2004).

Hasta el año 2009, sólo se había encontrado a L. villaregalis en dos localidades, una de ellas en un paredón ubicado al sur ("El Espinazo") donde las poblaciones crecen en un solo punto y la otra donde se observó en manchones de unos cuantos individuos dispersos a lo largo de una cañada localizada al norte, cerca del pueblo La Venta del Astillero ("La Venta"), ambas en el APFFLP (Hernández-López et al., 2009) (fig. 2, C y D). La vegetación circundante a las localidades donde se ubica $L$. villaregalis es bosque de pino-encino, siendo el clima predominante templado subhúmedo y semicálido subhúmedo del tipo estacional, conformado por dos periodos: estiaje (seco) que ocurre desde octubre hasta junio y el de lluvias (húmedo) en los meses restantes (CONANP, 2000). 


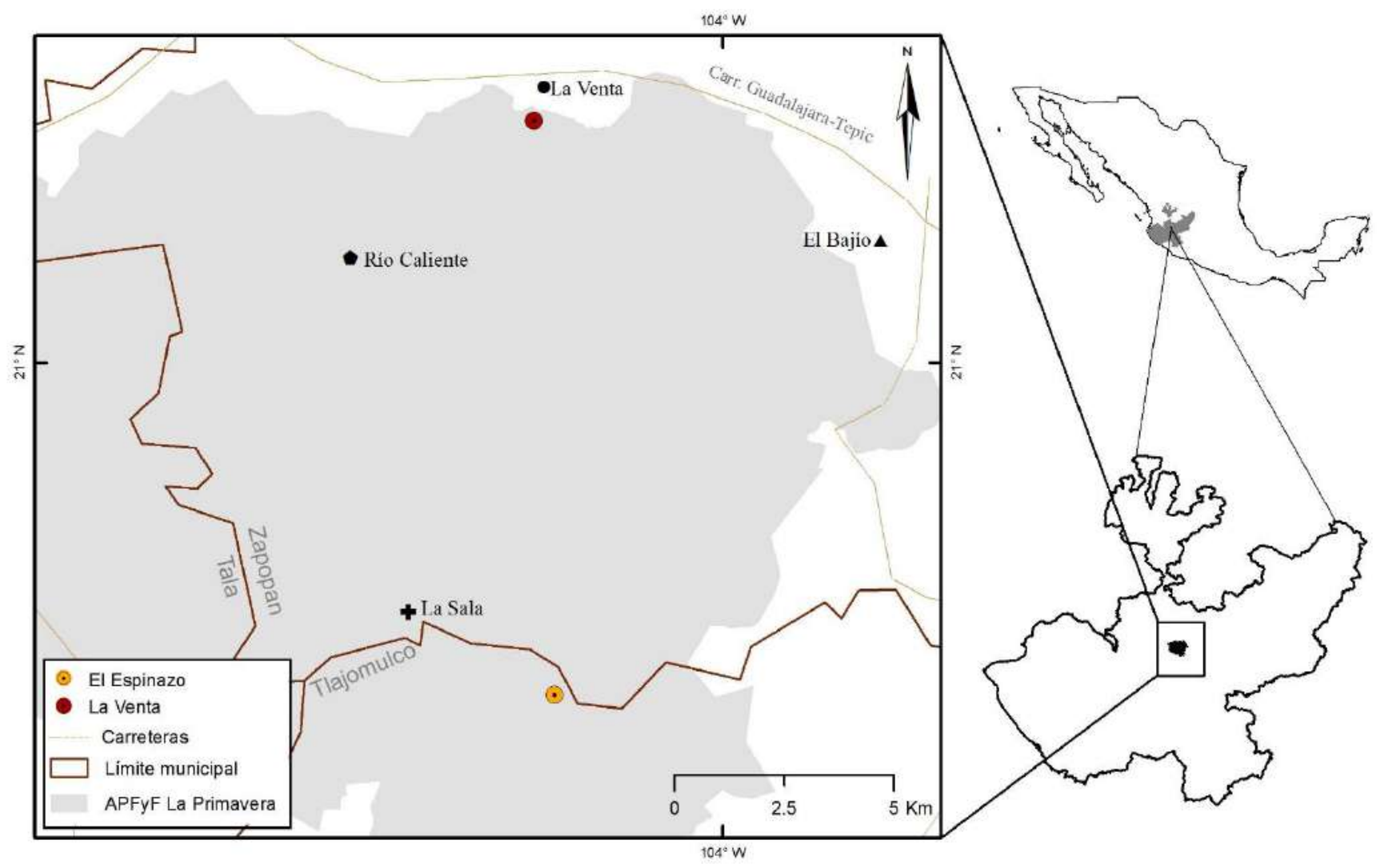

Fig. 1. El área natural protegida se ubica al oeste de la Zona Metropolitana de Guadalajara.

\section{Muestreo de suelo}

Características del suelo

Durante la época de estiaje del año 2010, en cada localidad donde se registró la presencia de $L$. villaregalis, se midió la profundidad del suelo alrededor de las raíces de 50 individuos. Por otra parte, tanto en "La Venta" como en "El Espinazo", se colectaron muestras de suelo ya sea cerca a las raíces de las plantas $(n=6)$ como de sitios donde la especie no estaba presente. Sin embargo, debido a que el suelo es poco profundo, las muestras de los diferentes sitios se tuvieron que mezclar conformando una sola (aproximadamente $500 \mathrm{~g}$ ), es decir, en total se obtuvieron 2 muestras por cada localidad (una del sitio donde crece la especie y otra donde no se observa). El suelo fue analizado en el Laboratorio de Agrología de la Universidad de Guadalajara, donde se realizaron las siguientes determinaciones: Textura (método de Bouyoucos), materia orgánica (método de Walkey-Black), $\mathrm{pH}$ mediante la relación 1:2 suelo:agua destilada, nitrógeno nítrico y nitrógeno amoniacal, fósforo, potasio, calcio, magnesio y manganeso (método de Morgan). Los resultados del análisis fueron interpretados con base en los criterios de calidad de suelo de SAGARPA y FAO (2012) y Castellanos et al. (2000).

\section{Asociación Micorrícica}

Con la finalidad de evaluar si las raíces de L. villaregalis establecen relación con hongos micorrícicos del suelo y en caso de haberla, si hay una potencial variación estacional de la colonización micorrícica, en cada una de las localidades se colectaron 5 plantas (con una altura aproximada de 15-20 cm y con hojas jóvenes) en dos épocas del año: la primera de ellas durante la fase de floración de la especie que ocurre en el estiaje, y la otra en la estación húmeda. Sin 
embargo, debido al bajo número de individuos en la localidad "La Venta", la comparación entre estaciones sólo se llevó a cabo en la localidad "El Espinazo".

Las plantas de L. villaregalis se llevaron al laboratorio, donde las raíces se limpiaron y almacenaron en alcohol (50\%); posteriormente, se procesaron siguiendo la técnica de tinción de Kormanik y McGraw (1982). Para cuantificar el porcentaje de colonización de las raíces por el hongo micorrícico (\%RC) se utilizó el método de intersección magnificada propuesto por McGonigle et al. (1990).

Análisis estadísticos

El análisis estadístico se realizó empleando el programa estadístico $\mathrm{R}$ versión 3.4.4 ( $R$ Core Team, 2018). Para evaluar si existen diferencias en la fertilidad del suelo entre sitios (donde la especie crece y donde está ausente) dentro de la misma localidad, se realizó una prueba de Mann-Withney pareada. Para evaluar las diferencias en \%RC entre las localidades se llevó a cabo una prueba de comparación de dos muestras (T-student). Asimismo, se determinó si existían diferencias entre el estiaje y la estación de lluvias utilizando la prueba T-student pareada en la localidad "El Espinazo".

Muestreo de vegetación

Para documentar la composición florística de las dos localidades donde crece L. villaregalis se realizaron salidas de campo entre diciembre del 2007 y marzo del 2010. El material recolectado se procesó con base en Lot y Chiang (1986) y se depositó en el Herbario Luz María Villareal de Puga de la Universidad de Guadalajara (IBUG). Ahí mismo, se llevó a cabo la determinación taxonómica de las muestras utilizando bibliografía especializada para posteriormente integrar un listado de las especies encontradas, ordenado alfabéticamente por nombre científico de los taxa y por presencia. Aparecen primero los taxa compartidos entre las dos localidades (Apéndice). En algunos casos, no fue posible determinar el material hasta el nivel de especie ya que al momento de la colecta no presentaba estructuras fértiles, por lo que en el listado sólo se indica el género y los datos del colector aparecen en blanco. Para analizar la presencia de malezas entre las especies registradas, se revisó la obra de Villaseñor y Espinosa-García (1998) y la plataforma de las malezas de México (CONABIO, 2019). Para describir las características fisonómicas de las localidades en "El Espinazo" se midió la longitud del paredón y la altura en tres puntos diferentes, mientras que en "La Venta" se definieron cinco puntos a lo largo de la cañada en los cuales se calculó el ancho y alto.

\section{REsultados}

Descripción del suelo

Se observó que L. villaregalis crece en suelos franco arenosos, poco profundos (promedio de $1.5 \mathrm{~cm}$ ) con un $\mathrm{pH}$ que oscila entre 4.5 y 5.5. Este tipo de suelo por su naturaleza arenosa es altamente permeable por lo que tienen poca retención de agua y nutrientes, así como baja inercia térmica (Castellanos et al., 2000). De acuerdo con SAGARPA y FAO (2012) y Castellanos et al. (2000) en ambas localidades, la planta crece en suelos con porcentajes bajos de agua aprovechable; niveles bajos de nitrógeno nítrico, nitrógeno amoniacal, calcio y magnesio; niveles medios de fósforo; y niveles altos y medio altos de potasio y manganeso (cuadro 1). En general, los valores de fertilidad entre ambas localidades son similares. Sin embargo, con excepción del nitrógeno y la materia orgánica, los niveles de nutrientes fueron más altos en "La Venta que en "El Espinazo". Desafortunadamente, dado el bajo número de replicas por localidad $(\mathrm{n}=1)$ no se puedo establecer si tales diferencias son estadísticamente significativas. Al comparar entre los sitios donde crece la planta y donde está ausente en cada una de las localidades no se observaron diferencias significativas $(\mathrm{p}>0.05)$. 
Cuadro 1. Características del suelo de los sitios donde hay presencia y ausencia de L. villaregalis dentro de las dos localidades del estudio. El valor de V y su probabilidad fueron calculados empleando la prueba Mann-Withney pareada. ND: no detectable.

\begin{tabular}{ccccccc}
\hline Variable & V & \multicolumn{2}{c}{ La Venta } & \multicolumn{2}{c}{ El Espinazo } & p \\
\hline & - & Presente & Ausente & Presente & Ausente & - \\
\hline Clase textural & - & $\mathrm{Fa}$ & $\mathrm{Fa}$ & $\mathrm{Fa}$ & Af & NA \\
\hline Agua aprovechable $\%$ & 0 & 13 & 12 & 9 & 6 & 0.5 \\
\hline Materia orgánica $\%$ & 2 & 1.88 & 2.26 & 4.24 & 2.19 & 0.9 \\
\hline $\mathrm{pH}$ & 0 & 5 & 5.5 & 4.5 & 4.7 & 0.5 \\
\hline Nitrógeno nítrico ppm & 0 & 0.5 & 1 & 1 & 1 & 0.9 \\
\hline Nitrógeno amoniacal ppm & 0 & 6 & 12 & 12 & 12 & 0.9 \\
\hline Fósforo ppm & 0 & 25 & 25 & 25 & 25 & 1 \\
\hline Potasio ppm & 0 & 250 & 250 & 250 & 250 & 1 \\
\hline Calcio ppm & 1 & 500 & 1200 & 500 & 500 & 0.9 \\
\hline Magnesio ppm & 0 & 25 & 25 & 12 & 12 & 0.9 \\
\hline Manganeso ppm & 3 & 8 & 5 & 2 & ND & 0.5 \\
\hline
\end{tabular}

Asociación micorrícica

Se encontró que $L$. villaregalis tiene una asociación micorrícica del tipo arbuscular, la cual se establece entre hongos del Phyllum Glomeromycota y el $80 \%$ de las familias de las plantas vasculares (Smith y Read, 2008). En este estudio, se registró un \%RC del $36.5 \pm 5.5$ en "La Venta" y $31.3 \pm 9.8$ en "El Espinazo" (cuadro 2), sin diferencia significativa entre localidades $\left(t_{8}=0.459, \mathrm{p}>0.05\right)$. Por otra parte, al comparar el \%RC entre diferentes épocas del año en la localidad de "El Espinazo" tampoco se detectaron diferencias significativas $\left(\mathrm{t}_{8}=0.303, \mathrm{p}>\right.$ 0.05 ; cuadro 3 ).

Cuadro 2. Porcentaje de colonización en la raíz de L. villaregalis por hongos micorrícicos arbusculares en cada localidad. Los valores representan el promedio y error estándar de la media $(\mathrm{n}=5)$. El valor de $\mathrm{t}$ y su probabilidad fueron calculados empleando la prueba T-student. RC: colonización total de las raíces.

\begin{tabular}{ccccc}
\hline Estructura & t & La Venta & El Espinazo & p \\
\hline Arbúsculo \% & 0.2 & $0.92 \pm 0.33$ & $5.35 \pm 3.37$ & 0.82 \\
\hline Vesículas \% & 0.2 & $5.30 \pm 1.15$ & $3.56 \pm 0.58$ & 0.83 \\
\hline Hifas \% & 0.3 & $30.29 \pm 4.42$ & $22.40 \pm 6.33$ & 0.74 \\
\hline RC \% & 0.6 & $36.52 \pm 5.51$ & $31.32 \pm 9.89$ & 0.53 \\
\hline
\end{tabular}


Cuadro 3. Porcentaje de colonización en la raíz de L. villaregalis por hongos micorrícicos arbusculares en la localidad "El Espinazo" en dos diferentes estaciones del año. Los valores representan el promedio y error estándar de la media $(\mathrm{n}=5)$. El valor de t y su probabilidad fueron calculados empleando la prueba T-student. RC: Colonización total de las raíces.

\begin{tabular}{ccccc}
\hline Estructura & $\mathbf{t}$ & Estiaje & Lluvias & p \\
\hline Arbúsculo \% & 0.6 & $5.35 \pm 3.37$ & $2.85 \pm 1.20$ & 0.58 \\
\hline Vesículas \% & -0.4 & $3.56 \pm 0.58$ & $4.04 \pm 1.07$ & 0.71 \\
\hline Hifas \% & -1.9 & $22.40 \pm 6.33$ & $41.98 \pm 5.16$ & 0.13 \\
\hline RC \% & -1.2 & $31.32 \pm 9.89$ & $48.88 \pm 5.10$ & 0.28 \\
\hline
\end{tabular}

\section{Descripción de la vegetación}

En el conjunto de las dos localidades estudiadas, se registró un total de 44 familias, 102 géneros y 140 taxa de plantas vasculares, incluyendo cinco categorías intraespecíficas (Apéndice). Asteraceae concentra la mayor riqueza de especies con 44 taxa $(32 \%$ del total), le siguen Poaceae y Pteridaceae con 6 y 5\% de las especies, respectivamente. Los géneros con mayor número de especies corresponden a Ageratina con siete y Pseudognaphalium con cinco, ambos se incluyen en Asteraceae. La forma biológica mejor representada es la herbácea con 102 taxa (73\% del total), le sigue la arbórea con $18(13 \%)$, la arbustiva con $16(11 \%)$ y sólo cuatro taxa son trepadoras. De las especies registradas, $22(16 \%)$ son consideradas como malezas, no obstante, sólo tres son de origen exótico o incierto. Entre las plantas no vasculares, se registra la presencia de musgo (Bryopsida) en las dos localidades, pero con mayor abundancia en "El Espinazo".

Respecto a la composición de especies, se encontró que 31 taxa (22.1\%) se comparten entre las dos localidades, de las cuales 19 son hierbas, 7 son árboles y el resto corresponden a arbustos y trepadoras. Se consideran malezas cinco de los taxa compartidos (Lobelia laxiflora, Lopezia racemosa, Oplismenus burmannii, Paspalum humboldtianum y Wigandia urens). La localidad de "La Venta" alberga 101 taxa (72\% del total) de los cuales $70(50 \%)$ son exclusivos y en "El Espinazo" se registran 70 (50\% del total), de ellos 39 (28\%) no se comparten (Apéndice). De las especies exclusivas de "La Venta" 84\% (59 taxa) corresponden a herbáceas que en su mayoría (64\%) pertenecen a la familia Asteraceae y a siete familias de helechos y afines. Se consideran malezas 14 taxa. Destacan tres especies de árboles; Clethra rosei, Morella cerifera y Oreopanax peltatus. Por otra parte, en "El Espinazo" los taxa exclusivos incluyen 24 hierbas $(61.5 \%)$ de las cuales casi la mitad forman parte de las Asteraceae. Las especies arbustivas y arbóreas conforman el restante $38.5 \%$. Sólo tres taxa exclusivos se reconocen como malezas. Entre los árboles se listan; Pinus douglasiana, P. maximinoi, Clethra hartwegii, Agarista mexicana var. pinetorum, Arbutus madrensis y Comarostaphylis glaucescens.

Las dos localidades donde crece L. villaregalis se encuentran en áreas de bosque de encino (Quercus) y bosque mixto de encino-pino, sin embargo, existen diferencias en la composición florística. A continuación, se describen las características de las dos localidades y la composición de la vegetación: La localidad de "La Venta" se ubica entre los 1530-1565 m s.n.m. donde $L$. villaregalis se encuentra en la parte alta y media de la cañada creciendo de forma dispersa a lo largo de un $\mathrm{km}$ del estrecho cañón con exposición noroeste, de forma que sólo recibe luz solar en el invierno durante algunas horas de la mañana. La cañada alcanza los $10.5 \mathrm{~m}$ en su parte más alta y en otras porciones no pasa de los $4 \mathrm{~m}$. Lo ancho varía entre 3 y $1.30 \mathrm{~m}$; la cañada se abre hacia la parte alta donde llega a los $7.5 \mathrm{~m}$ (fig. 2D). En el fondo de esta cañada los árboles son escasos y se trata de especies características de lugares húmedos 
como Fraxinus uhdei, Morella cerifera, Oreopanax peltatus y Prunus serotina. En el estrato arbustivo se observan Ageratina areolaris, A. dolichobasis, Cestrum nitidum, Hyptis albida y Cunila pycnantha. El estrato herbáceo que cubre las paredes se compone de algunos helechos y afines como Asplenium monanthes, Thelypteris rudis, Cheilanthes angustifolia, Adiantum capillus-veneris y Selaginella pallescens. También se encuentran algunos pastos como Muhlenbergia tenella, M. brevifolia, Oplismenus burmannii y Paspalum humboldtianum; además de Begonia gracilis, Drymaria gracillima, Moussonia elegans, Ageratina malacolepis y Heuchera orizabensis.

Respecto a la localidad "El Espinazo", esta se localiza entre los 2060-2080 m s.n.m. y es un paredón con exposición noroeste que debe su existencia a la apertura de un camino que asciende al cerro Planillas donde se localiza una torre de vigilancia. Presenta en forma general, la misma composición de suelo y rocas que la localidad de "La Venta", pero la población de $L$. villaregalis es quizá 20 veces más numerosa aquí y concentrada en una superficie más reducida, creciendo principalmente en la parte media y alta del paredón. Éste mide $50 \mathrm{~m}$ de largo y su altura es irregular alcanzando $17 \mathrm{~m}$ en la parte más alta, disminuyendo hasta los $3 \mathrm{~m}$ (fig. 2C). Es una pared casi vertical que en el invierno recibe poca radiación solar directa en las mañanas, el resto del día está bajo sombra dada en parte por otro paredón más pequeño ubicado frente a éste y por los árboles y arbustos que la coronan o que crecen entre la población de Lobelia. Los árboles que se encuentran adyacentes a esta población de L. villaregalis son: Clethra hartwegii, Comarostaphylis glaucescens, Pinus devoniana, P. oocarpa, P. maximinoi, Quercus resinosa y $Q$. viminea. Entre los arbustos se registran a Calliandra hirsuta, Monnina schlechtendaliana, Gaultheria erecta, Vaccinium stenophyllum y Wigandia urens. En cuanto a las herbáceas sobresalen Dalea roseiflora, Cerastium brachypodon, Salvia lavanduloides, Heterocentron mexicanum, Lopezia racemosa, Heuchera orizabensis, Bessera elegans y los pastos Aegopogon cenchroides, Paspalum humboldtianum y Oplismenus burmannii. En esta zona es frecuente observar especies arbóreas de los géneros Clethra, Pinus y Prunus y arbustivas como Wigandia creciendo en oquedades del paredón. No obstante, la mayoría no llega a establecerse y permanecen por poco tiempo debido a la erosión del suelo, mientras que las especies herbáceas tienen más éxito.

Cabe mencionar que L. villaregalis se encuentra en comunidades típicas de cañadas húmedas, por lo menos como sucede en las cañadas que forman parte del APFFLP. Las especies que comparten las condiciones ambientales en suelo y humedad entre las dos localidades son 31 , algunas se nombran a continuación: Fraxinus uhdei, Prunus serotina, Pinus devoniana, $P$. oocarpa, Quercus coccolobifolia, $Q$. resinosa y $Q$. viminea entre las arbóreas, y de las herbáceas Lopezia racemosa, Heuchera orizabensis, Paspalum humboldtianum, Oplismenus burmannii, Lobelia laxiflora y Moussonia elegans, entre otras (Apéndice). 

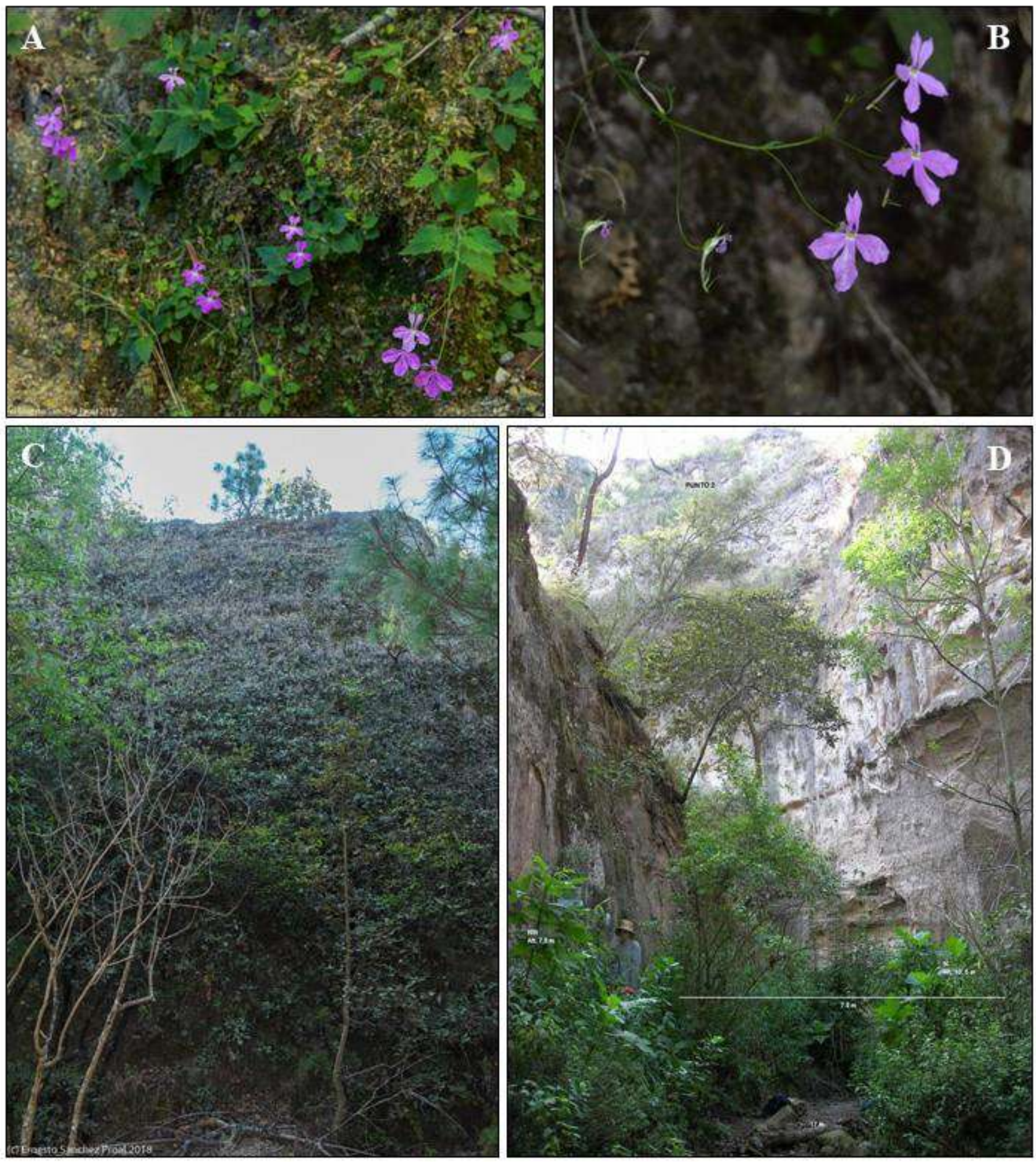

Fig. 2. A y B. Lobelia villaregalis en flor; C. Localidad El Espinazo; D. Localidad La Venta. Fotografías de E. Sánchez Proal (A y C), D. Figueroa García (B) y M. Harker (D). 


\section{DISCUSIÓN}

Ante la constante modificación de hábitats por razones antropogénicas, la generación de información sobre las características del hábitat donde las especies se desarrollan resulta urgente para su conservación, particularmente para aquellas que son endémicas restringidas. Las especies con distribuciones restringidas evolutivamente se han especializado con base en uno o más factores ambientales (Boisson et al., 2017; Irl et al., 2015). Esta especialización, las hace vulnerables a cambios en su hábitat y por lo tanto son especies prioritarias para la conservación (Niskanen et al., 2019). En este estudio, se generó información sobre algunos de los factores que conforman el hábitat donde se desarrolla L. villaregalis dentro del APFFLP, la cual se encuentra amenazada por actividades derivadas del crecimiento urbano de la Zona Metropolitana de Guadalajara.

Los resultados muestran que la especie se desarrolla en suelos con niveles bajos de nutrientes dada su alta permeabilidad debido a su naturaleza arenosa lo cual es característico de los suelos tipo regosol del APFFLP (CONANP, 2000). El regosol es un tipo de suelo joven presente en el $18.5 \%$ del territorio del país (SEMARNAT, 2002) principalmente en las regiones biogeográficas de la Sierra Madre Occidental, Eje Neovolcánico Transversal y Sierra Madre del Sur (SEMARNAT, 1998). El no encontrar diferencias significativas entre sitios donde $L$. villaregalis está presente y ausente, sugieren que la cañada y el paredón no presentan microhábitats definidos por características edáficas. Sin embargo, la baja retención de humedad del suelo, la presencia de taxa de sitios húmedos y la baja exposición solar de las localidades, indican que otros factores, por ejemplo, porcentaje de humedad relativa o cantidad de radiación solar podrían estar siendo de alguna manera determinantes en la distribución de la especie. Lo anterior contrasta con otros estudios donde se ha demostrado que el suelo es el factor que más influye en la diversidad vegetal (Huerta-Martínez et al., 2014, 2012). Asimismo, estos resultados sugieren que $L$. villaregalis podría estar presente en otras cañadas húmedas dentro del APFFLP y potencialmente en otras zonas montañosas del país, aunque debido al relieve geológico único del APFFLP (González Torreros et al., 2018) que pudo haber llevado a procesos de especiación y dispersión particulares al área, la probabilidad de que la especie se encuentre fuera del área protegida sea baja.

En ambas localidades, L. villaregalis estableció una asociación micorrícica del tipo arbuscular, aunque en un bajo \%RC comparada con otras especies (Olivera-Morales et al., 2011; Montañez Orozco et al., 2010). De la misma manera, al parecer se mantiene el \%RC, a pesar del cambio de estaciones durante el año como se pudo constatar con las plantas colectadas en "El Espinazo". La estabilidad en la asociación micorrícica entre la época de estiaje y lluvias no es inusual, ya que se ha observado en otras especies como Coccothrinax readii Quero (Polanco et al., 2013) y Agave angustifolia Haw (Ochoa-Meza et al., 2009). Por un lado, se menciona que una estrategia para enfrentar la limitación por nutrientes y agua sería el establecimiento de relaciones micorrícicas (Smith y Read, 2008; Ruiz-Lozano, 2003), por otra parte, una alta colonización en suelos pobres podría convertirse en una desventaja, ya sea por un alto gasto metabólico de la planta al tener que retribuir con carbono a los hongos micorrícicos (Lendenmann et al., 2011; Lynch y Ho, 2005; Nielsen et al., 1998); o a que la planta y el hongo micorrícico compiten por nutrientes (Johnson et al., 2015). Sin embargo, el hecho de haber reportado que $L$. villaregalis presenta asociación micorrícica del tipo arbuscular es un paso importante en el conocimiento de la biología de esta planta, donde el identificar las especies de hongos micorrícicos que se asocian, así como diseñar experimentos para probar su función y contribución en el desarrollo y reproducción de la planta son apremiantes.

En cuanto a la composición de la vegetación acompañante, es de esperarse que Asteraceae sea la familia con mayor riqueza de especies en las dos localidades, ya que es un grupo altamente representado en toda el área protegida (CONANP, 2000) y en otras áreas con bosques templados en Jalisco (García-Martínez y Rodríguez, 2018; Harker et al., 2017). No obstante, los sitios estudiados mostraron diferencias tanto en la riqueza como en la composición de 
especies, lo cual puede explicarse por el proceso de formación de ambas localidades. En la localidad "El Espinazo" el paredón que ocupa L. villaregalis se formó por la fragmentación de un cerro para construir un camino angosto en el año de 1972 (A. Curiel, comunicación personal). Posteriormente, se reforestó con especies de pino a lo largo del camino construido de modo que, actualmente la vía es estrecha y los árboles han alcanzado cerca de los $10 \mathrm{~m}$. Este hecho contrasta con la localidad de "La Venta", donde al parecer la cañada se ha formado con el paso del tiempo y por los procesos naturales. Otra diferencia fundamental entre las localidades es la superficie que ocupan, ya que como se mencionó, "El Espinazo" mide apenas $50 \mathrm{~m}$ de largo, mientras que "La Venta" alcanza un km de longitud, aproximadamente.

En "La Venta" habitan cuatro especies de orquídeas y 13 helechos exclusivos, lo que aunado a la presencia de Oreopanax peltatus y Morella cerifera, entre otras, reflejan un estado más conservado de esta localidad. No obstante que alberga el mayor número de especies arvenses (14), su abundancia es baja. En tanto que en "El Espinazo" sus especies exclusivas se distribuyen en un mayor número de familias y el número de malezas exclusivas es menor ( 3 de 8 presentes) que en "La Venta". Sin embargo, aquí se observan con mayor abundancia a Lobelia laxiflora, Oplismenus burmannii, Paspalum humboltianum, Baccharis salicifolia, Jaegeria hirta y Wigandia urens las cuales representan especies pioneras que han colonizado el sitio como se ha encontrado en otros bosques mixtos de pino-encino donde miembros de Asteraceae y Poaceae conforman principalmente el contingente de pioneras (Ramírez-Marcial et al., 1992).

Por otra parte, dado que el origen de "El Espinazo" fue una perturbación que implicó el desmonte y la apertura de material parental, puede decirse que L. villaregalis consigue colonizar sitios que, aunque han recibido cierto disturbio, con el tiempo en ellos llegan a crearse microhábitats (paredes con sustrato arenoso, permanentemente húmedas y con poca llegada de radiación solar) que favorecen a la planta. En este caso a $L$. villaregalis le tomó cerca de 50 años colonizar y establecerse exitosamente en "El Espinazo". No obstante, eso fue posible gracias a la baja incidencia de perturbación en el sitio en el periodo posterior a la apertura del camino, lo cual se evidencia en parte por la acumulación de una gruesa capa de combustible en el suelo (ausencia de incendios) y la presencia de fauna como Crotalus basiliscus, especie endémica de México y protegida bajo la Norma Oficial Mexicana 059 de la SEMARNAT.

Por tanto, los resultados sugieren que L. villaregalis tiene preferencia por lugares húmedos (posiblemente a causa de desarrollarse en suelos con poca retención de agua) y en buen estado de conservación. Actualmente, ambas poblaciones de L. villaregalis están expuestas al impacto antropogénico, ya sea por su cercanía a asentamientos humanos irregulares o senderos recreativos por lo que es necesaria una regulación del paso de transeúntes, evitar cambios ilegales del uso de suelo y realizar actividades de prevención de incendios para la prevención de daños en ambas poblaciones. Se pretende que los datos generados en esta investigación sean de utilidad para la exploración de nuevas localidades potenciales, así como la realización de estudios ecológicos para orientar la promoción y generación de programas para la conservación y repoblación asistida de L. villaregalis. Estas iniciativas de investigación y acciones de manejo son urgentes, ya que los sitios de estudio aquí documentados, fueron impactados por los incendios forestales que se registraron en el APFFLP en la temporada de estiaje del 2019 y por tal motivo, la permanencia de L. villaregalis en esos sitios es ahora incierta.

\section{CONCLUSIONES}

L. villaregalis se desarrolla en suelos franco arenosos, pobres, de poca profundidad y alta permeabilidad en los que establece asociaciones micorrícicas del tipo arbuscular, cuyo porcentaje de colonización se mantiene constante entre estaciones climáticas. La flora acompañante de esta especie y las condiciones generales de las localidades estudiadas reflejan su preferencia por hábitats húmedos y paredes o laderas pronunciadas con sustrato arenoso en 
buen estado de conservación. Las estrategias para la conservación de la especie, por tanto, deberán incluir acciones que eviten el deterioro de su hábitat. Asimismo, se sugiere que en las estrategias de propagación de esta especie se consideren la incorporación de microorganismos nativos del suelo (hongos micorrícicos) donde crece para aumentar su probabilidad de supervivencia.

\section{AGRADECIMiEnTos}

Se agradece al laboratorio de Agrología del CUCBA de la Universidad de Guadalajara por los análisis de suelos. Al Laboratorio Nacional de Identificación y Caracterización Vegetal (LaniVeg), Unidad Biogeografía, por la elaboración del mapa. A Darío Figueroa y Miguel A. García por la edición de las imágenes. Los créditos de las fotografías se anotan en las figuras correspondientes. Alejandro Muñoz Urías apoyó en la medición de una de las localidades. A Sofia Monroy y Pilar Zamora por informarnos sobre la localidad del Espinazo. A Eduardo Villa, Ana Molgora, Ana Galaviz y J.G. González por asistencia en campo. A Miguel A. Muñiz Castro por la revisión y sugerencias al documento. Al editor, Rafael Fernández Nava y a tres revisores anónimos quienes contribuyeron a la mejora del manuscrito.

\section{LITERATURA CITADA}

Ayers, T. J. (1987). Four species from western Mexico new to Lobelia (Campanulaceae: Lobelioideae). Brittonia, 34, 39:417. https://doi.org/10.2307/2807317

Boisson, S., Faucon, M. P., Le Stradic, S., Lange, B., Verbruggen, N., Garin, O., ... Mahy, G. (2017). Specialized edaphic niches of threatened copper endemic plant species in the D.R. Congo: implications for ex situ conservation. Plant and Soil, 413, 261. https://doi.org/10.1007/s11104-016-3095-7

Brady, N. C., y Weil, R. R. (2002). The nature and properties of soils (13th ed.). USA: Prentice Hall. $960 \mathrm{p}$.

Castellanos, J. Z., Uvalle-Bueno, J. X., y Aguilar-Santelises, A. (2000). Manual de interpretación de análisis de suelos y aguas. (Colección del Instituto de Capacitación para la Productividad Agrícola (INCAPA) (2nd ed.). México D.F. 226 p.

CONABIO. (2019). Malezas de México. Recuperado de http://www.conabio.gob.mx/malezasdemexico/2inicio/home-malezas-mexico.htm. Consultado el 15 de marzo de 2019

CONANP. (2000). Programa de Manejo del Area de Protección de Flora y Fauna La Primavera, México. México D.F.: CONANP-SEMARNAT. 132 p.

Curiel, A. (1988). Plan de Manejo Bosque La Primavera. Guadalajara, Jalisco.: Facultad de Agricultura. DICSA-Universidad de Guadalajara. $164 \mathrm{p}$.

Davidson, B. E., Novak, S. J., y Serpe, M. . (2016). Consequences of inoculation with native arbuscular mycorrhizal fungi for root colonization and survival of Artemisia tridentata ssp. wyomingensis seedlings after transplanting. Mycorrhiza, 26(6), 595-608. https://doi.org/10.1007/s00572-016-0696-1 LK

Gallegos, R. A. (Comp. (2011). El Bosque La Primavera y la Universidad de Guadalajara: una antología de biodiversidad y ciencia. Guadalajara, Jalisco.: Universidad de Guadalajara. $223 \mathrm{p}$.

García-Martínez, M. A., y Rodríguez, A. (2018). Vegetación y flora fanerogámica del área natural protegida Piedras Bola, Jalisco, México. Polibotánica, 46, 71-90. https://doi.org/10.18387/polibotanica.46.4

González Torreros, L., Valdivia Ornelas, L., y Gómez Sención, J. H. (2018). El contexto del territorio: una forma de introducción a la Sierra La Primavera. En L. González Torreros, L. Valdivia Ornelas, y J. H. Gómez Sención (Eds.), Diversidad Volcánica y Geopatrimonio en la Sierra La Primavera (pp. 9-42). Guadalajara, Jalisco.: Universidad de Guadalajara. Recuperado en 15 de Marzo de 2009 de 
http://www.publicaciones.cucsh.udg.mx/kiosko/2018/diversidad_volcanica.pdf

Gutiérrez-Sánchez, R. I., Castro-Castro, A., González-Gallegos, J. G., López-Enríquez, I. L., y Frías-Castro, A. (2018). Synopsis of the spurred species of Lobelia section Stenotium (Campanulaceae) in Sierra Madre Occidental, Mexico, and the description of two new species. Phytotaxa, 338(1), 33-48. https://doi.org/10.11646/phytotaxa.338.1.3

Harker, M., Hernández-López, L., Reynoso Dueñas, J. J., Gónzalez-Villarreal, L. M., Cedano Maldonado, M., Arias García, J. A., ... Quintero Fuentes, V. (2017). Actualización de la flora vascular de San Sebastián del Oeste, Jalisco, México. Ibugana, 8, 3-63.

Hernández-López, L., Harker, M., y Ramírez-Delgadillo, R. (2009). Nuevas localidades de Lobelia villaregalis (Campanulaceae: Lobelioideae), especie endémica de Jalisco. En S. Carvajal y E. Pimienta Barrios (Eds.), 2008 Avances en la Investigación en el CUCBA, XIX Semana Nacional de La Investigación Científica: (pp. 213-215). Zapopan, Jalisco: CUCBA, Universidad de Guadalajara.

Huerta-Martínez, F. M., Briones-Tirado, J. E., Neri-Luna, C., Muñoz-Urias, A., y Rosas Espinoza, V. C. (2014). Relaciones entre comunidades arbóreas, suelo y el gradiente altitudinal en el Volcán de Tequila, Jalisco. Revista Mexicana de Ciencias Forestales, 5(24), 202-215.

Huerta-Martínez, F. M., Muñoz-Urias, A., Neri-Luna, C., Uribe-Mu, C. A., y García-Moya, E. (2012). Reciprocal effects of plant-soil relationships at two spatial scales in a semiarid land of central Mexico. Journal of Agricultural Science and Technology, 2, 71-79.

Irl, S. D. H., Harter, D. E. V, Steinbauer, M. J., Gallego Puyol, D., Fernández-Palacios, J. M., Jentsch, A., y Beierkuhnlein, C. (2015). Climate vs. topography - spatial patterns of plant species diversity and endemism on a high-elevation island. Journal of Ecology, 103(6), 1621-1633. https://doi.org/10.1111/1365-2745.12463

Johnson, N. C., Wilson, G. W. T., Wilson, J. A., Miller, R. M., y Bowker, M. A. (2015). Mycorrhizal phenotypes and the Law of the Minimum. New Phytologist, 205(4), 1473-1484. https://doi.org/10.1111/nph.13172

Kormanik, P. P., y McGraw, A. C. (1982). Quantification of vesicular-arbuscular mycorrhizae in plant roots. En N. C. Schenck (Ed.), Methods and principles for mycorrhyzal research (pp. 37). St. Paul: Minnesota: American Phytopathological Society.

Lammers, T. G. (2011). Revision of the Infrageneric Classification of Lobelia L. (Campanulaceae: Lobelioideae). Annals of the Missouri Botanical Garden, 98(1), 3762. https://doi.org/10.3417/2007150

Lendenmann, M., Thonar, C., Barnard, R. L., Salmon, Y., Werner, R. A., Frossard, E., y Jansa, J. (2011). Symbiont identity matters: carbon and phosphorus fluxes between Medicago truncatula and different arbuscular mycorrhizal fungi. Mycorrhiza, 21(8), 689-702. https://doi.org/10.1007/s00572-011-0371-5

Lot, A., y Chiang, F. (1986). Manual de Herbario. Administración, Manejo de Colecciones, Técnicas de Recolección y Preparación de Ejemplares Botánicos. México, D.F.: Consejo Nacional de la Flora de México. $142 \mathrm{p}$.

Lynch, J. P., y Ho, M. D. (2005). Rhizoeconomics: Carbon costs of phosphorus acquisition. Plant and Soil, 269, 45-56. https://doi.org/10.1007/s11104-004-1096-4

McGonigle, T. P., Miller, M. H., Evans, D. G., Fairchild, G. L., y Swan, J. A. (1990). A new method which gives an objective measure of colonization of roots by vesiculararbuscular mycorrhizal fungi. New Phytologist, 115(3), 495-501. https://doi.org/10.1111/j.1469-8137.1990.tb00476.x

Montañez Orozco, I., Vargas Sarmiento, C., Cabezas Gutiérrez, M., y Cuervo Andrade, J. (2010). Colonización micorrícica en plantas de aguacate (Persea americana L.). Revista U.D.C.A Actualidad y Divulgación Científica, 13(2), 51-60. Recuperado en 8 de septiembre de 2019 de http://www.scielo.org.co/pdf/rudca/v13n2/v13n2a07.pdf

Neri-Luna, C., y Villarreal-Ruiz, L. (2012). Simbiosis micorrícica: un análisis de su relevante función ecosistémica y en la provisión de servicios ambientales. En F.M. HuertaMartínez y L. P. Castro-Félix (Eds.), Interacciones Ecológicas (pp. 37-62). Universidad de Guadalajara.

Nielsen, K., Bouma, T. J., Lynch, J. P., y Eissenstat, D. M. (1998). Effects of phosphorus 
availability and vesicular-arbuscular mycorrhizas on the carbon budget of common bean (Phaseolus vulgaris). New Phytologist, 139(4), 647-656. https://doi.org/10.1046/j.1469-8137.1998.00242.x

Niskanen, A. K. J., Niittynen, P., Aalto, J., Väre, H., y Luoto, M. (2019). Lost at high latitudes: Arctic and endemic plants under threat as climate warms. Diversity and Distributions, 25(5), 809-821. https://doi.org/10.1111/ddi.12889

Ochoa-Meza, A., Esqueda, M., Fernández-Valle, R., y Herrera-Peraza, R. (2009). Variación estacional de hongos micorrízicos arbusculares asociados con Agave angustifolia Haw. en la Sierra Sonorense, México. Revista Fitotecnia Mexicana, 32(3), 189-199. $\begin{array}{llllll}\text { Recuperado en } & 8 & \text { de } & \text { septiembre de }\end{array}$ http://www.scielo.org.mx/scielo.php?script=sci_arttextypid=S018773802009000300006 ynrm=iso

Olivera-Morales, D., Castillo-Argüero, S., Guadarrama, P., Ramos-Zapata, J., Álvarez-Sánchez, J., y Hernández-Cuevas, L. (2011). Establecimiento de plántulas de Quercus rugosa Née inoculadas con hongos micorrizógenos arbusculares en un bosque templado de México. Boletín de La Sociedad Botánica de México, (89), 115-121. Recuperado en 8 de septiembre de 2019 de http://www.scielo.org.mx/scielo.php?script=sci_arttextypid=S036621282011000200007 ylng=esytlng=es.

Polanco, G., Carrillo, L., Espadas, C., Reyes-García, C., Guadarrama, P., y Orellana, R. (2013). Asociación micorrízica arbuscular en Coccothrinax readii Quero. Tropical and Subtropical Agroecosystems, 16(2), 223-233. Recuperado en 8 de septiembre de 2019 https://www.redalyc.org/articulo.oa?id=93928324011

R Core Team. (2018). R: A language and environment for statistical computing. R. Foundation for Statistical Computing. Recuperado de http://www.r-project.org/

Ramírez-Marcial, N., González-Espinoza, M., y Quintana, P. F. (1992). Banco y lluvia de semillas en comunidades sucesionales de bosque de pino-encino de los Altos de Chiapas, México. Acta Botánica Mexicana, (20), 59-75. Recuperado en 8 de septiembre de https://www.redalyc.org/articulo.oa?id=57402009

Reyna Bustos, O. F. (1989). Estudio de la Vegetación de la reserva Forestal de La Primavera, Jalisco. Tesis. Facultad de Agricultura. Universidad de Guadalajara.Guadalajara, Jalisco, México. 70 p.

Reyna Bustos, O. F. (2004). Arboles y arbustos del Bosque La Primavera. Guía ilustrada. Guadalajara, Jalisco.: Universidad de Guadalajara-Comisión Nacional para el Conocimiento y Uso de la Biodiversidad.118 p.

Ruiz-Lozano, J. (2003). Arbuscular mycorrhizal symbiosis and alleviation of osmotic stress. New perspectives for molecular studies. Mycorrhiza, 13(6), 309-317. https://doi.org/10.1007/s00572-003-0237-6

Rzedowski, J. (2006). Vegetación de México. México, D.F.: Comisión Nacional para el Conocimiento y Uso de la Biodiversidad. $504 \mathrm{p}$.

Rzedowski, J. (2019). Inventario actualizado de las especies mexicanas de la familia $\begin{array}{llll}\text { Campanulaceae. } & \text { Botanical } & \text { Sciences, } & \text { 97(1), }\end{array}$ https://doi.org/http://dx.doi.org/10.17129/botsci.2085

SAGARPA, y FAO. (2012). Línea de Base del Programa de Sustentabilidad de los Recursos Naturales Componente: Línea de base del uso sustentable del suelo para el año 2010. México, D.F. Recuperado en 3 de febrero de 2019 de https://www.faoevaluacion.org.mx/rn/ind_fin/suelos/documento_metodologico_suelos.pdf

SEMARNAT. (1998). Mapa de suelos dominantes de lä República Mexicana. Recuperado de http://www.conabio.gob.mx/informacion/metadata/gis/edafo4mgw.xml?_httpcache=ye s\&_xsl=/db/metadata/xsl/fgdc_html.xsl\&_indent=no. Consultado el 25 de septiembre de $\overline{2} 019$.

SEMARNAT. (2002). Informe de la situación del medio ambiente en México. Recuperado de http://www.paot.org.mx/centro/inesemarnat/informe02/estadisticas_2000/informe_2000/index.htm Consultado el 25 de septiembre 2019 


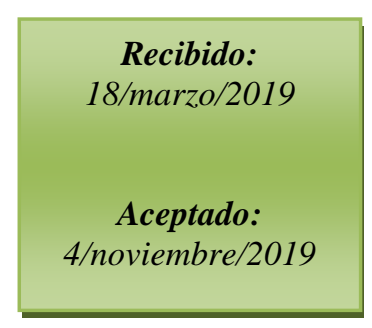

Smith, S. E., y Read, D. J. (2008). Mycorrhyzal symbiosis (3rd ed.). Academic Press. 800 p.

Valdivia Ornelas, L. (2018). Caracterización de los procesos geológico-geomorfológicos y clasificación del relieve de la Sierra La Primavera. En L. González Torreros, L. Valdivia Ornelas, y J. H. Gómez Sención (Eds.), Diversidad Volcánica y Geopatrimonio en la Sierra La Primavera (pp. 43-136). Guadalajara, Jalisco.: Universidad de Guadalajara. Recuperado en 5 de Marzo de 2019 de http://www.publicaciones.cucsh.udg.mx/kiosko/2018/diversidad_volcanica.pdf

Villaseñor, J. L., y Espinosa-García, F. J. (1998). Catálogo de malezas de México. México, D.F.: Universidad Nacional Autónoma de México. Consejo Nacional Consultivo Fitosanitario y Fondo de Cultura Económica. 448 p.

Wiens, J. J., y Donoghue, M. J. (2004). Historical biogeography, ecology and species richness.

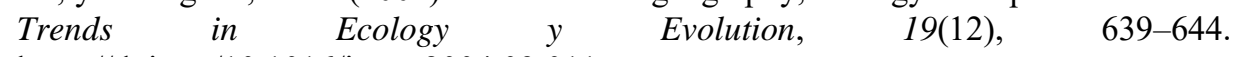
https://doi.org/10.1016/j.tree.2004.09.011 


\section{Apéndice de la flora asociada a Lobelia villaregalis en el Área Natural Protegida Bosque La Primavera.}

Se marca con una $\mathrm{x}$ la presencia de los taxa en las dos localidades. Se listan primero las especies compartidas. Los nombres de los colectores (Cols.) se abrevian como: MH (Mollie Harker), LHL (Leticia Hernández López), EV (Edith Villa), LMVP (Luz María Villareal de Puga) y TA (Tina Ayers). La forma biológica de las plantas vasculares: $\mathrm{H}$ - herbácea; a -arbustiva; A - arbórea; y $\mathrm{T}$ - trepadora. Ejemplares sin colector significa que fueron observadas.

\begin{tabular}{|c|c|c|c|c|}
\hline Taxa & $\begin{array}{c}\text { Forma } \\
\text { biológica }\end{array}$ & La Venta & El Espinazo & Cols. y núm. de colecta \\
\hline Adiantum poiretii Wikstr. & $\mathrm{H}$ & $\mathrm{x}$ & $\mathrm{x}$ & LHL 1037, MH 4123 \\
\hline Agave guadalajarana Trel. & $\mathrm{a}$ & $\mathrm{x}$ & $\mathrm{x}$ & MH 4134 \\
\hline $\begin{array}{l}\text { Ageratina adenophora (Spreng.) R. M. } \\
\text { King \& H. Rob. }\end{array}$ & $\mathrm{H}$ & $\mathrm{x}$ & $\mathrm{x}$ & EV 25, MH 4141 \\
\hline $\begin{array}{l}\text { Ageratina dolichobasis (McVaugh) R. M. } \\
\text { King \& H.Rob. }\end{array}$ & a & $\mathrm{x}$ & $\mathrm{x}$ & MH 4143, 4128 \\
\hline Cheilanthes angustifolia Kunth & $\mathrm{H}$ & $\mathrm{x}$ & $\mathrm{x}$ & EV 22, MH 4122 \\
\hline Clematis acapulcensis Hook. \& Arn. & $\mathrm{T}$ & $\mathrm{x}$ & $\mathrm{x}$ & \\
\hline Critoniopsis foliosa (Benth.) H. Rob. & $\mathrm{H}$ & $\mathrm{x}$ & $\mathrm{x}$ & MH 3771, LH 953 \\
\hline Fraxinus uhdei (Wenzig) Lingelsh. & A & $\mathrm{x}$ & $\mathrm{X}$ & \\
\hline $\begin{array}{l}\text { Gyrandra tenuifolia (M. Martens \& } \\
\text { Galeotti) Mansion }\end{array}$ & $\mathrm{H}$ & $\mathrm{x}$ & $\mathrm{x}$ & $\begin{array}{l}\text { LHL 993, 1006, MH } \\
3773\end{array}$ \\
\hline Helianthemum glomeratum (Lag.) Lag. & $\mathrm{H}$ & $\mathrm{X}$ & $\mathrm{x}$ & \\
\hline Heterocentron mexicanus Hook. \& Arn. & $\mathrm{H}$ & $\mathrm{x}$ & $\mathrm{X}$ & \\
\hline $\begin{array}{l}\text { Heterotheca inuloides Cass. var. rosei } \\
\text { Wagenknecht }\end{array}$ & $\mathrm{H}$ & $\mathrm{x}$ & $\mathrm{x}$ & MH 3772 \\
\hline Heuchera orizabensis Hemsl. & $\mathrm{H}$ & $\mathrm{x}$ & $\mathrm{x}$ & LHL 948, 996 \\
\hline Hieracium abscissum Less. & $\mathrm{H}$ & $\mathrm{x}$ & $\mathrm{x}$ & \\
\hline Hyptis albida Kunth & $\mathrm{a}$ & $\mathrm{x}$ & $\mathrm{X}$ & \\
\hline Lobelia laxiflora Kunth & $\mathrm{H}$ & $\mathrm{x}$ & $\mathrm{x}$ & \\
\hline Lobelia villaregalis Ayers & $\mathrm{H}$ & $\mathrm{x}$ & $\mathrm{x}$ & $\begin{array}{l}\text { LMVP 2463; TA 596; } \\
\text { EV 17; LHL 936, 954 }\end{array}$ \\
\hline Lopezia racemosa Cav. & $\mathrm{H}$ & $\mathrm{x}$ & $\mathrm{X}$ & LHL 938 \\
\hline Moussonia elegans Decne. & $\mathrm{H}$ & $\mathrm{x}$ & $\mathrm{x}$ & \\
\hline Muhlenbergia brevifolia Scribn. ex Beal & $\mathrm{H}$ & $\mathrm{X}$ & $\mathrm{X}$ & MH 4133 \\
\hline Oplismenus burmannii (Retz.) P. Beauv. & $\mathrm{H}$ & $\mathrm{x}$ & $\mathrm{x}$ & \\
\hline Oxypappus scaber Benth. & $\mathrm{H}$ & $\mathrm{x}$ & $\mathrm{x}$ & MH 3758, 4120 \\
\hline Paspalum humboldtianum Flügge & $\mathrm{H}$ & $\mathrm{x}$ & $\mathrm{x}$ & \\
\hline Pinguicula parviflora B. L. Rob. & $\mathrm{H}$ & $\mathrm{x}$ & $\mathrm{X}$ & MH 3962 \\
\hline Pinus devoniana Lindl. & $\mathrm{A}$ & $\mathrm{x}$ & $\mathrm{x}$ & \\
\hline Pinus oocarpa Schiede ex Schlecht. & A & $\mathrm{x}$ & $\mathrm{x}$ & EV 19 \\
\hline Prunus serotina Ehrh. & A & $\mathrm{x}$ & $\mathrm{x}$ & \\
\hline Quercus coccolobifolia Trel. & A & $\mathrm{x}$ & $\mathrm{x}$ & \\
\hline Quercus resinosa Liebm. & A & $\mathrm{X}$ & $\mathrm{x}$ & \\
\hline Quercus viminea Trel. & A & $\mathrm{x}$ & $\mathrm{x}$ & \\
\hline Wigandia urens (Ruiz \& Pav.) Kunth & $\mathrm{a}$ & $\mathrm{x}$ & $\mathrm{x}$ & \\
\hline $\begin{array}{l}\text { Acmella radicans (Jacq.) R. K. Jansen var. } \\
\text { radicans }\end{array}$ & $\mathrm{H}$ & $\mathrm{x}$ & & MH 4091 \\
\hline Acourtia sp. & $\mathrm{H}$ & $\mathrm{x}$ & & \\
\hline $\begin{array}{l}\text { Adenophyllum cancellatum (Cass.) } \\
\text { Villarreal }\end{array}$ & $\mathrm{H}$ & $\mathrm{x}$ & & MH 4094 \\
\hline Adiantum capillus-veneris $\mathrm{L}$. & $\mathrm{H}$ & $\mathrm{x}$ & & LHL 1035, MH 4136 \\
\hline Adiantum concinnum Humb. \& Bonpl. & $\mathrm{H}$ & $\mathrm{X}$ & & LHL 945 \\
\hline
\end{tabular}




\begin{tabular}{|c|c|c|c|c|}
\hline Taxa & $\begin{array}{c}\text { Forma } \\
\text { biológica }\end{array}$ & La Venta & El Espinazo & Cols. y núm. de colecta \\
\hline Ageratina areolaris (DC.) Gage & $\mathrm{a}$ & $\mathrm{x}$ & & MH 3754 \\
\hline $\begin{array}{l}\text { Ageratina malacolepis (B. L. Rob.) R. M. } \\
\text { King \& H. Rob. }\end{array}$ & $\mathrm{H}$ & $\mathrm{x}$ & & MH 4142 \\
\hline $\begin{array}{l}\text { Ageratina muelleri (Klatt) R. M. King \& } \\
\text { H. Rob. }\end{array}$ & $\mathrm{H}$ & $\mathrm{x}$ & & MH 4092 \\
\hline Asplenium monanthes $\mathrm{L}$. & $\mathrm{H}$ & $\mathrm{x}$ & & LHL 1014,944 \\
\hline Asplenium sp. & $\mathrm{H}$ & $\mathrm{x}$ & & LHL 943 \\
\hline Aster subulatus Michx. & $\mathrm{H}$ & $\mathrm{x}$ & & MH 4093 \\
\hline Begonia gracilis Kunth & $\mathrm{H}$ & $\mathrm{x}$ & & LHL 1003 \\
\hline $\begin{array}{l}\text { Bidens bigelovii A. Gray var. angustifolia } \\
\text { (DC.) Ballard }\end{array}$ & $\mathrm{H}$ & $\mathrm{x}$ & & MH 4138 \\
\hline Blechnum occidentale L. & $\mathrm{H}$ & $\mathrm{x}$ & & LHL 1012 \\
\hline Bletia reflexa Lindl. & $\mathrm{H}$ & $\mathrm{x}$ & & LHL 947 \\
\hline Bletia roezlii Rchb. f. & $\mathrm{H}$ & $\mathrm{x}$ & & \\
\hline Carpochaeta grahamii A. Gray & $\mathrm{H}$ & $\mathrm{x}$ & & MH 3766 \\
\hline Cestrum nitidum M. Martens \& Galeotti & $\mathrm{a}$ & $\mathrm{x}$ & & LHL 949 \\
\hline $\begin{array}{l}\text { Chromolaena ovaliflora (Hook. \& Arn.) } \\
\text { R. M. King \& H. Rob. }\end{array}$ & $\mathrm{a}$ & $\mathrm{x}$ & & LHL 950 \\
\hline Clethra rosei Britt. & A & $\mathrm{x}$ & & \\
\hline Canavalia villosa Benth. & $\mathrm{T}$ & $\mathrm{x}$ & & LHL 1002 \\
\hline $\begin{array}{l}\text { Conyza canadensis (L.) Cronquist var. } \\
\text { canadensis }\end{array}$ & $\mathrm{H}$ & $\mathrm{x}$ & & MH 4089 \\
\hline $\begin{array}{l}\text { Cosmos landii Sherff var. achalconensis } \\
\text { Melchert }\end{array}$ & $\mathrm{H}$ & $\mathrm{x}$ & & MH 4088, 4124 \\
\hline Cunila pycnantha Rob. \& Greenm. & $\mathrm{a}$ & $\mathrm{x}$ & & LHL 946 \\
\hline Cystopteris fragilis (L.) Bernh. & $\mathrm{H}$ & $\mathrm{x}$ & & LHL 1038 \\
\hline Desmodium angustifolium (H. B. K.) DC. & $\mathrm{H}$ & $\mathrm{x}$ & & LHL 1004 \\
\hline Drymaria gracillima Rose & $\mathrm{H}$ & $\mathrm{x}$ & & LHL 1016 \\
\hline $\begin{array}{l}\text { Dryopteris maxonii Underw. C. Chr. ex C. } \\
\text { Chr. }\end{array}$ & $\mathrm{H}$ & $\mathrm{x}$ & & LHL 1040 \\
\hline Erigeron karvinskianus DC. & $\mathrm{H}$ & $\mathrm{x}$ & & MH 3775a \\
\hline Euphorbia nutans (Lag.) Small & $\mathrm{H}$ & $\mathrm{x}$ & & LHL 1032 \\
\hline Euphorbia ocymoidea L. & $\mathrm{H}$ & $\mathrm{x}$ & & LHL 1019,1033 \\
\hline Galinsoga parviflora Cav. & $\mathrm{H}$ & $\mathrm{x}$ & & MH 4137 \\
\hline Guardiola sp. & $\mathrm{H}$ & $\mathrm{x}$ & & \\
\hline Habenaria jaliscana $\mathrm{S}$. Watson & $\mathrm{H}$ & $\mathrm{x}$ & & \\
\hline Hyptis mutabilis (Rich.) Brig. & $\mathrm{H}$ & $\mathrm{x}$ & & LHL 1043 \\
\hline $\begin{array}{l}\text { Iresine diffusa var. diffusa Humb. \& } \\
\text { Bonpl ex Willd. }\end{array}$ & $\mathrm{H}$ & $\mathrm{x}$ & & LHL 952 \\
\hline Lopezia miniata Lag. ex DC. & $\mathrm{H}$ & $\mathrm{x}$ & & \\
\hline Loeselia glandulosa (Cav.) G. Don & $\mathrm{H}$ & $\mathrm{x}$ & & LHL 1018 \\
\hline Loeselia mexicana (Lam.) Brand & $\mathrm{H}$ & $\mathrm{x}$ & & LHL 1001 \\
\hline Malaxis unifolia Michx. & $\mathrm{H}$ & $\mathrm{x}$ & & \\
\hline Morella cerifera (L.) Small & A & $\mathrm{x}$ & & MH 3774 \\
\hline Muhlenbergia rigida (Kunth) Kunth & $\mathrm{H}$ & $\mathrm{x}$ & & MH 4134 \\
\hline Muhlenbergia tenella (H.B.K.) Trin. & $\mathrm{H}$ & $\mathrm{x}$ & & LHL 1034 \\
\hline Nemastylis tenuis (Herb.) S. Watson & $\mathrm{H}$ & $\mathrm{x}$ & & \\
\hline Oenothera rosea L' Her. ex Ait. & $\mathrm{H}$ & $\mathrm{x}$ & & \\
\hline Oreopanax peltatus Linden ex Regel & A & $\mathrm{x}$ & & \\
\hline Pellaea ternifolia (Cav.) Link & $\mathrm{H}$ & $\mathrm{x}$ & & LHL 1036 \\
\hline Physalis pubescens L. & $\mathrm{H}$ & $\mathrm{x}$ & & LHL 1015 \\
\hline
\end{tabular}




\begin{tabular}{|c|c|c|c|c|}
\hline Taxa & $\begin{array}{c}\text { Forma } \\
\text { biológica }\end{array}$ & La Venta & El Espinazo & Cols. y núm. de colecta \\
\hline Pitcairnia palmeri S. Watson & $\mathrm{H}$ & $\mathrm{x}$ & & MH 3961 \\
\hline Pityrogramma ebenea (L.) Proctor & $\mathrm{H}$ & $\mathrm{x}$ & & MH 4135 \\
\hline Polygala berlandieri $\mathrm{S}$. Watson & $\mathrm{H}$ & $\mathrm{x}$ & & LHL 1018 \\
\hline Prochnyanthes mexicana Zucc. & $\mathrm{H}$ & $\mathrm{x}$ & & \\
\hline $\begin{array}{l}\text { Pseudognaphalium attenuatum (DC.) A. } \\
\text { Anderb. }\end{array}$ & $\mathrm{H}$ & $\mathrm{x}$ & & MH 4095 \\
\hline $\begin{array}{l}\text { Pseudognaphalium chartaceum (Greenm.) } \\
\text { A. Anderb. }\end{array}$ & $\mathrm{H}$ & $\mathrm{x}$ & & MH 4090 \\
\hline $\begin{array}{l}\text { Pseudognaphalium inornatum (DC.) A. } \\
\text { Anderb. }\end{array}$ & $\mathrm{H}$ & $\mathrm{x}$ & & MH 3761 \\
\hline $\begin{array}{l}\text { Pseudognaphalium oxyphyllum (DC.) } \\
\text { Kirp. }\end{array}$ & $\mathrm{H}$ & $\mathrm{x}$ & & MH 4140 \\
\hline $\begin{array}{l}\text { Pseudognaphalium semiaplexicaule (DC.) } \\
\text { A. Aderb. }\end{array}$ & $\mathrm{H}$ & $\mathrm{x}$ & & MH 4139 \\
\hline Quercus obtusata Humb. \& Bonpl. & A & $\mathrm{x}$ & & \\
\hline Selaginella pallescens (C. Presl) Spring & $\mathrm{H}$ & $\mathrm{x}$ & & LHL 1005 \\
\hline Selaginella sp. & $\mathrm{H}$ & $\mathrm{x}$ & & LHL 941 \\
\hline $\begin{array}{l}\text { Solanum nigrescens M. Martens \& } \\
\text { Galeotti }\end{array}$ & $\mathrm{H}$ & $\mathrm{x}$ & & LHL 1036 \\
\hline Stevia jaliscensis B. L. Rob. & $\mathrm{H}$ & $\mathrm{x}$ & & MH 3760 \\
\hline Stevia origanoides Kunth & $\mathrm{H}$ & $\mathrm{x}$ & & MH 3752 \\
\hline Stevia ovata Willd. & $\mathrm{H}$ & $\mathrm{x}$ & & \\
\hline Thelypteris rudis (Kunze) Proctor & $\mathrm{H}$ & $\mathrm{x}$ & & LHL 1042 \\
\hline $\begin{array}{l}\text { Toxicodendron radicans (L.) Kuntze } \\
\text { subsp. divaricatum (Greene) Gillis }\end{array}$ & $\mathrm{T}$ & $\mathrm{x}$ & & \\
\hline Verbesina cinerascens B. L. Rob. & $\mathrm{H}$ & $\mathrm{x}$ & & LHL 951, MH 3762 \\
\hline Viguiera pringlei B. L. Rob. \& Greenm. & $\mathrm{H}$ & $\mathrm{x}$ & & \\
\hline Vitis sp. & $\mathrm{T}$ & $\mathrm{x}$ & & \\
\hline $\begin{array}{l}\text { Woodwardia spinulosa M. Martens \& } \\
\text { Galeotti }\end{array}$ & $\mathrm{H}$ & $\mathrm{x}$ & & LHL 1013 \\
\hline Aegopogon cenchroides Humb. \& Bonpl. & $\mathrm{H}$ & & $\mathrm{x}$ & \\
\hline $\begin{array}{l}\text { Agarista mexicana (Hemsl.) Judd. var. } \\
\text { pinetorum (Standl. \& L. O Williams) Judd }\end{array}$ & A & & $\mathrm{x}$ & MH 4129 \\
\hline $\begin{array}{l}\text { Ageratina blepharilepis (Sch. Bip.) R. M. } \\
\text { King \& H. Rob. }\end{array}$ & $\mathrm{H}$ & & $\mathrm{x}$ & EV 24, MH 4126 \\
\hline $\begin{array}{l}\text { Ageratina choricephala (B. L. Rob.) R. M. } \\
\text { King \& H. Rob. }\end{array}$ & $\mathrm{H}$ & & $\mathrm{x}$ & EV 26 \\
\hline Ageratum corymbosum Zucc. & $\mathrm{a}$ & & $\mathrm{x}$ & \\
\hline Arbutus madrensis S. González-Elizondo & A & & $\mathrm{x}$ & MH 4130 \\
\hline Aristida scribneriana Hitchc. & $\mathrm{H}$ & & $\mathrm{x}$ & \\
\hline Baccharis salicifolia (Ruiz \& Pav.) Pers. & $\mathrm{a}$ & & $\mathrm{x}$ & \\
\hline Bessera elegans Schult $\mathrm{f}$. & $\mathrm{H}$ & & $\mathrm{x}$ & \\
\hline $\begin{array}{l}\text { Brickellia pedunculosa (DC.) Harcombe } \\
\& \text { Beaman }\end{array}$ & $\mathrm{H}$ & & $\mathrm{x}$ & MH 4127 \\
\hline Buddleja sp. & $\mathrm{a}$ & & $\mathrm{x}$ & \\
\hline Calliandra hirsuta (G. Don) Benth. & $\mathrm{a}$ & & $\mathrm{x}$ & LHL 998 \\
\hline $\begin{array}{l}\text { Cerastium brachypodon (Engelm.) B. L. } \\
\text { Rob. }\end{array}$ & $\mathrm{H}$ & & $\mathrm{x}$ & \\
\hline $\begin{array}{l}\text { Comarostaphylis glaucescens (Kunth) } \\
\text { Zucc. }\end{array}$ & A & & $\mathrm{x}$ & \\
\hline Cheilanthes kaulfussii Kunze & $\mathrm{H}$ & & $\mathrm{x}$ & MH 4121 \\
\hline
\end{tabular}




\begin{tabular}{|c|c|c|c|c|}
\hline Taxa & $\begin{array}{c}\text { Forma } \\
\text { biológica }\end{array}$ & La Venta & EI Espinazo & Cols. y núm. de colecta \\
\hline Clethra hartwegii Britt. & A & & $\mathrm{x}$ & LHL 992 \\
\hline Dalea roseiflora (Rydb.) Riley & $\mathrm{H}$ & & $\mathrm{x}$ & LHL 999 \\
\hline Erigeron velutipes Hook. \& Arn. & $\mathrm{H}$ & & $\mathrm{x}$ & EV 23 \\
\hline Gaultheria erecta Ventanat & $\mathrm{a}$ & & $\mathrm{x}$ & EV 21 \\
\hline Hypericum glomeratum Lag. & $\mathrm{H}$ & & $\mathrm{x}$ & \\
\hline Jaegeria hirta (Lag.) Less. & $\mathrm{H}$ & & $\mathrm{x}$ & MH 4119 \\
\hline Lasianthaea sp. & $\mathrm{H}$ & & $\mathrm{x}$ & \\
\hline Monnina schlechtendaliana D. Dietr. & $\mathrm{a}$ & & $\mathrm{x}$ & LHL 994 \\
\hline Muhlenbergia watsoniana Hitchc. & $\mathrm{H}$ & & $\mathrm{x}$ & \\
\hline Oncidium brachyandrum Lindl. & $\mathrm{H}$ & & $\mathrm{x}$ & \\
\hline Pteridium aquilinium (L.) Kuhn & $\mathrm{H}$ & & $\mathrm{x}$ & \\
\hline Pinus douglasiana Martínez & A & & $\mathrm{x}$ & EV 20 \\
\hline Pinus maximinoi $\mathrm{H}$. E. Moore & $\mathrm{A}$ & & $\mathrm{x}$ & LHL 997 \\
\hline Pyrrhopappus sp. & $\mathrm{H}$ & & $\mathrm{x}$ & \\
\hline Salix bonplandiana Kunth & A & & $\mathrm{x}$ & \\
\hline Salvia lavanduloides Kunth & $\mathrm{H}$ & & $\mathrm{x}$ & MH 4131 \\
\hline Senecio stoechadiformis DC. & $\mathrm{H}$ & & $\mathrm{x}$ & \\
\hline Sisyrinchium сеrnиит (Bickn.) Kearney & $\mathrm{H}$ & & $\mathrm{x}$ & LHL 995 \\
\hline Tephrosia watsonii (Standl.) Macbr. & $\mathrm{H}$ & & $\mathrm{x}$ & \\
\hline Trachypogon secundus (Presl) Scribn. & $\mathrm{H}$ & & $\mathrm{x}$ & MH 3902 \\
\hline Vaccinium stenophyllum Steud. & $\mathrm{a}$ & & $\mathrm{x}$ & \\
\hline $\begin{array}{l}\text { Verbesina fastigiata B. L. Rob. \& } \\
\text { Greenm. }\end{array}$ & $a$ & & $\mathrm{x}$ & \\
\hline Verbesina sphaerocephala A.Gray & $\mathrm{H}$ & & $\mathrm{x}$ & \\
\hline Viguiera ensifolia (Sch. Bip.) S. F. Blake & $\mathrm{H}$ & & $\mathrm{x}$ & MH 4132 \\
\hline
\end{tabular}

